\title{
Mediatización Urbana. Nuevas Dinámicas de Comportamiento entre Individuo, Medios y Territorio
}

\author{
Urban Mediatization. New Dynamics of Behavior between Individual, Media \\ and Territory
}

\section{Mediatização urbana. Novas Dinâmicas de Comportamento entre Indivíduo, Mídia e Território}

\author{
Otniel Josafat López Altamirano \\ Arquitecto, Ph.D en Design \\ Universidad Autónoma "Benito Juárez" de Oaxaca, México \\ otniel_digital@hotmail.com \\ (iD) https://orcid.org/0000-0001-7593-9715 \\ Wendy Margarita Montes Ponce \\ Ph.D. en Arquitectura. \\ Universidad Autónoma "Benito Juárez" de Oaxaca, México \\ wmontes.cat@uabjo.mx \\ (iD) https://orcid.org/0000-0003-1962-4676 \\ Esteban Sumano Sánchez \\ Ph.D. en Arquitectura. \\ Universidad Autónoma Benito Juárez de Oaxaca, México \\ es261253@hotmail.com \\ (iD) https://orcid.org/0000-0001-9852-0474
}

Recibido: mayo 1 de 2019

Aceptado: junio 30 de 2020

Publicado: Julio 10 de 2020

\section{RESUMEN}

Se discuten las recientes dinámicas de comportamiento entre Individuo-Medios y Territorio, lo cual, en el momento histórico que sobrevivimos, resulta muy pertinente para desdoblar aquellos tópicos que requieren ser analizados a través de lentes transdisciplinares, de tal modo que se puedan reconocer los fenómenos que intervienen, enriquecen, y a la vez afectan la forma en que habitamos, nos constituimos como seres humanos y las identidades que conforman nuestro tejido social.

Palabras clave: Medios; ciudad; territorio; transmedia.

\section{ABSTRACT}

The recent dynamics of behavior between Individual-Media and Territory are discussed, which, in the historical moment that we survive, is very pertinent to unfold those topics that need to be analyzed through transdisciplinary lenses, in such a away that can be recognized the phenomena that intervene, enrich and at the same time affect the way we inhabit, we constitute ourselves as human beings and the identities that make up our social fabric.

Keywords: Media; city; territory; transmedia.

Cómo citar (APA)

López-Altamirano, O.J., Montes-Ponce, W.M., Sumano-Sánchez, E. (2020). Mediatización Urbana. Nuevas Dinámicas de Comportamiento entre Individuo, Medios y Territorio. Procesos Urbanos. 7(2):e494. https://doi.org/10.21892/2422085X.494 


\section{RESUMO}

Discute-se a recente dinâmica de comportamento entre Indivíduo-Mídia e Território, que, no momento histórico em que vivemos, é muito pertinente desdobrar aqueles temas que precisam ser analisados através de lentes transdisciplinares, para reconhecer os fenómenos que intervêm, enriquecem e ao mesmo tempo afetam o modo como habitamos, nos constituímos como seres humanos e as identidades que compõem nosso tecido social.

Palavras-chave: Mídia; cidade; território; transmídia

\section{INTRODUCCIÓN}

Los medios de comunicación como la radio, la televisión, el teléfono, la fotografía, la literatura o el cine, al igual que las plataformas de reciente generación, desarrollaron mecanismos que trajeron un alto impacto sobre el comportamiento del individuo en relación con su contexto y sus realidades. Cada uno de los medios han sido clasificados por teóricos en comunicación como McLuhan (1996) y Levinson (2015). Esto, conforme a su grado de complejidad, resolución y el nivel de interacción que alcanzan los medios entre hombrehombre y hombre-máquina.

Estas conjugaciones de tareas entre medios e individuos crean un cruce de mensajes, que ofrecen otras posibilidades de comunicación e interpretación para las audiencias. A partir de eso, podemos raciocinar al hibridismo, que refiere a una temática de descubrimiento y creación. Según McLuhan (1964, p.67) "los medios son agentes productores de conocimientos y la combinación o hibridismo de estos agentes (...) ofrece una oportunidad especialmente favorable para la observación de sus componentes y propiedades estructurales".

Un parteaguas en el desarrollo tecnológico del siglo presente es la internet. A partir de la democratización de esta, las telecomunicaciones trazaron líneas de infraestructura, así como de equipamiento urbano. Este hecho permitió el uso del servicio que, muy pronto, se convertiría en una red global, la cual soportaría gran parte del desarrollo comercial, cultural, social e ideológico de diversos territorios. Por lo que se plantea una nueva manera de pensar la Internet, una que da origen a un mundo mucho más smart de lo que imaginamos, dice Martel (2105), así también, a la diversidad de las Internets, las singularidades nacionales, lingüísticas, culturales que tienen su debido lugar en el mundo digital.
Aunado a lo arriba mencionado, las implementaciones tecnológicas han sido parte del desarrollo e identidad de diversos centros urbanos, manteniendo estos una relación con los medios análogos y con los medios que ya se basan en sistemas de geolocalización en beneficio del comercio, locomoción, socialización, habitabilidad, alimentación, entretenimiento, salud, contingencia, educación y trabajo, por mencionar tan solo algunas de las actividades que las plataformas mediáticas están reconfigurando durante la última década. Especialmente en estos momentos de crisis, en que la pandemia global ocasionada por el virus el Sars- CoV- 2 (Covid-19) interfiere en el desarrollo humano, como hemos podido ser testigos en gran parte de las ciudades, donde las plataformas de comunicación convergen y son los espacios para la interacción, educación, trabajo y socialización.

Por ello, se seleccionaron una serie de temáticas que motivan a la investigación y discusión, tanto a profesionales como a académicos, por medio de las cuales compartan los resultados de sus investigaciones, reflexiones sobre las implementaciones, los impactos, las problemáticas $y$, de igual forma, las responsabilidades que las nuevas plataformas de comunicación mediática y sus contenidos líquidos están revolucionando sobre las dinámicas de comportamiento humano en los diferentes territorios y centros urbanos.

Algunos gobiernos de países emergentes, que enfrentan grandes desafíos junto a otras economías con las que comparten una serie de rasgos, como gran población, vasto territorio y una destacada participación en el comercio internacional, hace que sus ejes de desarrollo apuesten por la proyección de ciudades inteligentes. Ya Martel (2015) se refería a Skolkovo, como la ciudad rusa que pretende ser inteligente, inaugurada en 2016 y en plena región gélida de la tundra, donde la temperatura llega a $30^{\circ} \mathrm{C}$ bajo cero. Algunos de estos modelos inteligentes, implementan centros de aprendizaje en negocios y comercio en el que 
se proponen ideas innovadoras soportadas por un modelo económico.

Estas mismas ciudades inteligentes, se configuran a partir de principios de orden progresista, siendo ecológicamente amigables, verdes y totalmente conectadas (De la Cruz y Guevara, 2015). Skolkovo además pretende responder a prioridades de modernización: eficacia energética, biomedicina, tecnologías nucleares y programa espacial de telecomunicaciones, todas ellas vinculadas a empresas de esos diferentes sectores, alrededor de un ecosistema en que conviven startups, investigadores, inversionistas y universidades, en otras palabras, una libre convivencia; todo eso desplantado sobre un régimen político, bastante cuestionable en temas de libertad de expresión, derechos humanos y libre tránsito de navegación web.

Desde esta perspectiva, dicen Cárdenas y Camargo (2016), las ciudades inteligentes son aquellas que han logrado articular y permitir el acceso a mejores servicios entre los ciudadanos que las habitan, trabajan y se recrean dentro de estas, mejorando el nivel de vida, gracias a que generan diferentes alternativas, basadas en la sostenibilidad, para realizar sus actividades cotidianas dirigidas a producciones en tecnología, arquitectura, diseño de productos, multimedia, moda y arte.

Lo cierto es, que este tipo de ciudades emergentes inteligentes son un prototipo de aldea interconectada: gran parte de su funcionamiento se basa en la 5G y la minería de datos. Este binomio garantiza un laboratorio experimental para el procesamiento de informaciones y establecer modelos funcionales que después serán incorporados en sistemas de mayor complejidad en grandes centros urbanos. Esto quiere decir, una ciudad que brinde mejores condiciones de vida para sus residentes y visitantes a través de los beneficios que trae el ancho de banda. El desarrollo tecnológico está acompañado de la ya constante discusión sobre la protección y manejo de datos de los ciudadanos digitales, puesto que tanto gobiernos como empresas privadas son las que proponen plataformas y servicios a partir del análisis big data para establecer patrones de comportamiento.

Sin duda, una ciudad inteligente resulta muy atractiva para los individuos familiarizados en esta brecha digital, aunque el habitar en una ciudad de este tipo, requiere que sus ciudadanos adopten un complejo concepto de vida, similar a seguir un instructivo que indique "como habitar en un ecosistema inteligente". En otras palabras, adiestrarse en una cibersociedad. Esta misma será marcada por las cuestiones y el concepto de la red, por la diseminación de la información y por el rápido desarrollo tecnológico de las plataformas, sistemas, interfaces, software y redes que se utilizan constantemente.

Sobre esta red, Moura (2003) dice que la misma presenta la posibilidad de un movimiento con una especie de interconectividad e intercomplejidad generalizada. $Y$ complementa con las palabras de Parente (1999, p. 78) quién determina que el "ciberespacio es el nuevo espacio de comunicación de la humanidad, aquel que integra algunas de las más importantes innovaciones en el campo de la electrónica, de la cibernética, de la computación, la información y la comunicación".

En esa misma perspectiva, el concepto de cibercultura, en la percepción de Rüdiger (2002, online), nace de la crítica ideológica, contando con los siguientes elementos: el neoliberalismo como un modelo político, la globalización determinada por la economía y la Internet como una red de intercambios. Por tanto, es una conjugación entre tecnología y la vida social en la cultura contemporánea, significando una reinvención cotidiana. Esto mismo, permite el surgimiento de un "movimiento social" con su grupo líder (la juventud metropolitana escolarizada), que crean comunidades virtuales a partir de la suma de las inteligencias colectivas.

Las diversas sociedades cibernéticas son de tipo transversal, son mutables, flexibles y restrictas a su vez; simbolizan las utopías en mundos paralelos. Las minorías forman sus propias comunidades, procurando estar alejadas de los sistemas que limitan sus acciones de comportamiento social, tal como sucede en los regímenes conservadores en Irán, China, Rusia, Corea del Norte, entre otras.

En contramano a las restricciones de orden social, son cada vez más los ejemplos de gobiernos que operan estrategias de cohesión social, a partir de modelos urbanísticos más integrales y complejos, dirigidos hacia la revitalización urbana, principalmente en centros metropolitanos $y$, en segundo orden, los espacios rurales. Las variables que determinan las acciones para la elaboración de proyectos de este tipo son de orden social, 
económico, cultural y de seguridad, que traen enormes beneficios a mediano y largo plazo, en una alta plusvalía de los espacios intervenidos, además de una fuerte carga de identidad.

Un proyecto que ilustra lo antes enunciado, fue "El Programa Mural de la Ciudad de las Artes en Philadelphia", que unió a artistas y comunidades a través de un proceso de colaboración, con raíces en las tradiciones de la pintura mural, para crear el arte que transforma los espacios públicos y las vidas individuales. Artistas que tuvieron la capacidad para colaborar con los profesionales del diseño, administradores de las artes y la comunidad (vecinos de los diferentes barrios intervenidos), que, además, tienen sus raíces en el deseo de encontrar un lenguaje común entre la vida cotidiana y las artes.

Del mismo modo en que ya habíamos reflexionado sobre este proyecto en la publicación "El Mural como vehículo narrativo e identidad ciudadana" en 2016, la ciudad de Philadelphia reconoce la trascendencia del proyecto "La Milla del Mural", ya que es palpable el trabajo multidisciplinario, entre profesionales de las artes y de diseño, benefactores que aportaron los recursos para llevar a cabo y todos aquellos que contribuyeron en la intervención en mayor o igual medida en cada una de las piezas. Además, el interés de los grupos ciudadanos concretó esta estrategia que obtuvo como resultado un compromiso simbólico entre individuo-proyecto-ciudad.

Otro exitoso proyecto de revitalización urbana ha sido el High Line Park en Manhattan, Nueva York, el mismo que responde al perfil cosmopolita de esta ciudad, en la que constantemente se reconfiguran los espacios habitacionales y de esparcimiento. Un parque de diseño, que rescata la identidad arquitectónica de un barrio dedicado a la industria en lado oeste de la isla. Si bien, Manhattan es una importante plataforma de desarrollo creativo e intelecto, arquitectos y paisajistas intervinieron en la propuesta de esta línea urbana vegetal. Consideramos que esta es una visita obligatoria para entender el concepto de urbanismo vertical y una experiencia inmersiva de la gran manzana.

"Desafortunadamente, el centro de Sao Paulo, por diversas razones, viene repeliendo a la gente. Y queremos un espacio público que atraiga a las personas", dice el artista Yung (2019, p. 2) refiriéndose al acuario urbano con un área que supera los 10.000 metros cuadrados, lo equivalente a dos campos y medio de fútbol. Cabe hacer mención que esta iniciativa es híbrida, ya que la población se aproxima al proyecto por medio de una aplicación digital con inmersión 3D y realidad virtual, misma que ofrece una "completa experiencia sensorial."

Como podemos observar, las acciones de revitalización de espacios públicos son tan amplios y ambiciosos como sean requeridos, es claro que el presupuesto público juega un factor importante en el alcance satisfactorio de este tipo de acupunturas urbanas. Por otro lado, la iniciativa privada también entra en esta partida, inyectando capital de forma directa o en sociedad con el gobierno para el fomento cultural. Algunas ciudades consiguen reestructurar su propia imagen a partir de nuevos modelos de configuración urbana, entrando a categorías que las eleva a ciudades de diseño, como lo son Londres, Nueva York o Vancouver. En ellas, se celebran los festivales internacionales de ciudades que impulsan el diseño socialmente responsable, considerando ejes formales como: habitantes, movilidad, identidad de la ciudad, medio ambiente, espacio público y economía creativa.

Algunos mandatarios han determinado, entre su agenda de desarrollo económico, apostar por los Medios y el Turismo, como sectores que contribuyen al soporte económico de algunas de sus comunidades, así como al posicionamiento de marcas nacionales, con énfasis en el aspecto visual, señala Bonsiepe (2011), presentándose de modo más atrayente en el ámbito internacional (...). Para obtener el máximo provecho, se recomienda encuadrar no solo a las empresas sino también a las ciudades, a las regiones y países. Aplicando las técnicas de branding, son generadas ventajas competitivas.

El mismo Bonsiepe (2011, p.60), dice que:

"el esfuerzo de ostentar una identidad inconfundible se manifiesta en la iniciativa de las Nuevas Ciudades de Diseño, presumiblemente diferentes de las ciudades de diseño establecidas, como Milán y Londres. Estas Nuevas Ciudades de Diseño exhiben a las llamadas creative industries para comprobar su carácter excepcional, a los cuales pertenecen los sectores de la industria cinematográfica, televisión, marketing, publicidad, investigaciones en tendencias, moda, publicaciones, arquitectura, diseño 
de exposiciones y eventos, diseño gráfico, diseño industrial y nueva media, vale decir los creative people".

La fuerte influencia persuasiva que generan las plataformas mediáticas sobre destinos que se vuelven turísticos, las podemos constatar en producciones audiovisuales de cine y televisión. La serie americana Sex and the City, transmitida por la cadena HBO en 1998, es una indiscutible semblanza de un "estilo de vida" en los Estados Unidos, donde todo ocurre apenas en los sitios más destacables de Manhattan. Quién también retrata la identidad de esta ciudad en un medio impreso, a través de sus maravillosas ilustraciones y con un estilo gráfico muy peculiar, es el ilustrador checo M. Sasek. "Esto es Nueva York" fue publicado en 1960 y continúa siendo reeditado; en él, se describen las características formales de esta metrópoli, su tráfico, sus historias, los periódicos de los domingos, el ferry de Staten Island, la Estatua de la Libertad, Greenwich Village, Times Square, el Rockefeller Center, Chinatown, el Central Park y toda la magia que envuelve a esta ciudad. Entre otras de sus creaciones destacan: Esto es Paris, Esto es Londres, Esto es Hong Kong, Esto es San Francisco y más.

Por su parte, Turquía se abrió al turismo internacional y durante el 2012 redujo los impuestos para las producciones audiovisuales extranjeras, abaratando los costos en rodajes cinematográficos. Así, parte del guion del Agente 007 en Skyfall, se filmó en las inmediaciones de El Gran Bazar de Estambul y un año más tarde volvería a ser el escenario para la película hollywoodense Water Diviner.

El atractivo masivo de ciertos centros urbanos es constantemente posicionado en las redes sociales, por medio de imágenes, comentarios y tendencias; "lo demuestran los tres hashtags de las ciudades más populares durante el 2016: \#NYC, \#London, \#Paris, las cuales fueron mencionadas en más de 180 millones de tweets", según lo comentan Gassmann; Böhm y Palmié (2019).

Todo asentamiento humano, cíclicamente requiere de reconfiguraciones en su infraestructura, equipamiento, espacios y servicios, ya que todo crecimiento demográfico y migración de sus habitantes intervienen en la morfología de las trazas urbanas. Los modelos pilotos iniciales, muchas de las veces son superados y las ramificaciones hacia el crecimiento habitacional entran en conflictos legales con los lineamientos constructivos de cada entidad. El tema de la Locomoción es una variable constante en los planes de desarrollo urbano. En la publicación "La ciudad como red y sistema de navegación" (2017) ya habíamos discutido que "los procesos conectivos son la base del tejido urbano; encontramos que uno de los componentes centrales del intelecto humano, es la capacidad de establecer conexiones, ideas que dan mejor comprensión de la naturaleza; esto sucede del mismo modo al establecer una analogía entre las conexiones mentales y las conexiones entre los elementos de una ciudad".

En el mismo documento arriba mencionado, también apuntamos que "las redes digitales, como la web, así como las redes urbanísticas son muy semejantes y consisten en la interacción de redes de conectividad en diversos niveles. Una trayectoria se establece por la unión de filamentos próximos de una red, igual que la necesidad de tener muchas alternativas de trayectorias y compararlas; esa podríamos decir que es la clave, igual como sucede con el pensamiento raciocinado".

En ese sentido, el avance tecnológico ha dado un salto bastante relevante en movilidad a partir de sistemas de geolocalización. Las aplicaciones móviles que brindan servicios de transporte conectan a los pasajeros con los conductores de vehículos registrados, ofreciendo precios competitivos; además de tener presencia global, este tipo de servicios ha permitido la autonomía de aquellos individuos que se desplazan constantemente o que optimizan sus rutas de navegación en centros urbanos, en los que no se cuenta con un sistema de transporte que satisfaga sus necesidades.

Son diversos los modelos de aplicaciones móviles. Algunas han sido procesadas en juzgados por el tema legal en el manejo de datos de sus usuarios, o bien, sus servicios han sido restringidos en algunos centros urbanos, tal es el caso de Uber en Barcelona o en Montreal. En ellas, los sindicatos de transporte privado manifestaron su descontento frente a una competencia (dicen ellos) desleal y ventajosa de esta empresa globalizada.

Bajo el mismo sistema de geolocalización, el servicio de entrega a domicilio ha significado una nueva categoría entre los establecimientos que operan mediante el envío de alimentos, 
e-commerce o farmacéutica. Estos modelos de comercio establecieron variables medibles que determinan la satisfacción del usuario y el posible éxito de la empresa en cuestión. Sin embargo, los algoritmos de estas plataformas se contraponen con elementos físicos de la topografía urbana, así como las condiciones de rendimiento de cada uno de los repartidores, algunos motorizados en el mejor de los casos. Falzoni (2020 online) apunta que "el cálculo de kilometraje recorrido es realizado por un algoritmo y quien pedalea en movilidad activa en la ciudad, sabe que tiene que rodar mucho más de lo que la aplicación sugiere, pues el repartidor intenta evitar los obstáculos urbanos".

Algoritmos, confidencialidad en el manejo de datos, zonificaciones urbanas, derechos laborales y redes de navegación, son los temas que se debaten en una sociedad hiperconectada que tiende cada vez más por el servicio de delivery.

Los sistemas de localización no solo benefician a las empresas, sino que también los datos producidos por usuarios a partir de su georreferencia, construyen un banco de informaciones aplicables en la prevención y atención frente a eventos de riesgo o catástrofes de cualquier tipo. Estos Medios actúan en momentos de contingencia, permitiendo, así, la instantánea información en momentos críticos. Por mencionar un ejemplo. "\#Monterreyfollow no es un individuo o una cuenta. No pertenece a nadie. Es una conversación, la cual permite que se comparta información de las zonas de gran violencia en ciudades donde el índice de homicidios es de los más elevados en México", dice Martel (2015 p.154). La cuenta se describe como "Una red de ciudadanos unidos por un \#MONTERREY más seguro \#MtyFollow \#AlertaMty \#AlertaNL \#RiesgoMty"1.

Los habitantes de la ciudad de Monterrey, México, pasaron a seguir en 2009 el hashtag \#Monterreyfollow, una palabra clave en Twitter. "Quedamos informados en tiempo real sobre dónde hay peligro, dónde está ocurriendo una matanza, dónde se encuentra un retén de policía, también cuales bares y restaurantes cuentan con seguridad", explica Carmen Junco, del Gobierno del Estado de Nuevo León, en entrevista para Martel (2015 p. 156). Teniendo lo anterior en mente, Johnson (2012 p. 68) interpreta a este proceso de comunicación como una heurística de sobrevivencia: "observar

1. https://twitter.com/monterreyfollow?lang=es si un amigo hombre de las cavernas come algunas frutas silvestres y este muere, hace que no quieras arriesgarte en descubrir si aquellas frutas matan personas. Esto genera, que no quieras comer más de esas frutas y decirles a tus amigos para que no las coman".

"En un contexto de violencia endémica, las redes sociales se revelan preciosas. Más allá de que sean plataformas americanas, Twitter y Facebook son usadas de maneras diferentes de un país a otro. "En zonas de narcotráfico, es frecuente que circulen mensajes muy sencillos, sin nombre ni apellido, del tipo: Hay nueve cuerpos en esta o aquella calle, o tres cuerpos fueron ahorcados en tal puente". Comenta el bloguero Antonio Martínez en entrevista para Martel (2015 p. 157).

La información disponible en cualquier red social es construida con base en la participación de los usuarios o seguidores, quienes comparten todo tipo de contenido, desde textual hasta audiovisual, que complemente el tópico del cual se discute. Como observamos en el ejemplo arriba mencionado, se concretó una amalgama funcional entre usuarios-ciudad-medio digital. En otras palabras, tecnologías de la información al servicio ciudadano en un escenario de contingencia, causado por la violencia. Respecto al servicio digital Gassmann, Böhm y Palmié (2019 p. 29) consideran que "las ciudades son una de las creaciones más complejas de la humanidad y, por lo tanto, el soporte digital es muy valioso. Todos los componentes que forman parte de este sistema ciberfísico mejoran la densidad de la sombra digital".

Estos medios se extienden como redes de manera transversal en todo asentamiento humano; el contenido es concreto, breve y prácticamente en tiempo real. Su objetivo es disponer elementos que permitan a los participantes la rápida toma de decisiones y reaccionar ante un evento social encuadrado en una traza urbana.

En ese mismo sentido, es que opera Kibera en Kenia, una de las más pobladas comunidades en el mundo, en extrema pobreza y que ha sido difícil de ubicar. Ya habíamos desdoblado parte de este proyecto en la publicación Transmedia Digital Inclusion (2018). Map Kibera fue desarrollado por una organización no lucrativa nombrada como Ushahidi, que significa "testigo" en swahili. De este modo, un grupo de voluntarios realizaron el levantamiento cartográfico de toda el área para 
su mapeo digital, el mismo que se encuentra disponible para su consulta en mapkibera.org; en él se pueden visualizar pasajes, caminos, puntos de agua bebible, baños públicos limpios, escuelas, iglesias y cibercafés.

La información en la plataforma es constantemente actualizada, es gratuita y colectiva. La colección masiva de datos es subministrada por el público y transferida a un sistema de geolocalización, la cual, después de ser verificada es subida. Actualmente, esta plataforma incluyó nuevas variables para su consulta y medición en temas referentes al Covid-19, tales como número de casos positivos confirmados; recursos, servicios y suministros; casos sospechosos de Covid-19; media, noticias e información.

Además de todo lo anterior descrito, las plataformas de comunicación virtual pueden llegar a intervenir en contextos sujetos a restricciones ideológicas, de género, credo y cualquier otra que contraponga a los Derechos Humanos. "Facebook facilita la constitución de redes de amigos extremadamente elásticas y adaptables, incluso en las situaciones de riesgo. Como es el caso de control policial argelino existente en Facebook (...) aunque el riesgo es menor que en un blog"; comenta Walid para Martel (2014, sección World Wide Gay), militante en Facebook, donde gestiona varias páginas gayfriendly.

Como podemos ir constatando, grupos de individuos se relacionan, interactúan, participan e intervienen en procesos sociales y de desarrollo, que afectan las dinámicas en sus comunidades y espacios geográficos. Por ello, algunos de estos individuos entienden la morfología, la configuración y las posibilidades que la ciudad ofrece como una plataforma de comunicación mediática. Sobre esto, en la publicación "La ciudad como red y sistema de navegación" (2016) decíamos, que "la navegación urbana siempre fue delimitada por trazos planimétricos, referencias, trayectos diseñados hacia la exploración e inquietud de los usuarios o habitantes, para el caso de las ciudades (...) con forme al desarrollo tecnológico el individuo continúa vinculándose con mayor facilidad para encontrarse con otros usuarios, formar parte de múltiples grupos, interactuar en ambientes dinámicos, de reposo, esparcimiento y entretenimiento, entre otros más".
Esto quiere decir, que la ciudad ofrece espacios, los cuales, la mayoría de las veces, han sido proyectados para que los habitantes recurran a ellos y puedan vivenciar cualquier tipo de experiencia, ya sea en plazas, parques, museos abiertos, ferias, mercados o ambientes naturales, por mencionar algunos.

Apoyados en lo anterior, ya discutíamos en el texto "Hombre Bestia entre las calles rosarinas" (2015, p. 146) sobre "la relación que existe entre el habitante como actor y la ciudad como el escenario, misma que plantea un enlace tremendamente estrecho al igual que fuerte entre estos dos entes, donde intervienen puentes históricos, sociales, culturales, políticos, económicos, etc. En otras palabras "No somos solo espectadores, sino actores que compartimos el escenario con todos los demás participantes. Nuestra percepción del medio ambiente no es continua, sino parcial y fragmentaria. Casi todos los sentidos entran en acción y la imagen es realmente una combinación de todos ellos".

Entonces, identificamos a la ciudad como el espacio material que alberga expresiones lúdicas, al igual que significativas para la población. Por ello, cada vez son más los proyectos que involucran a las tecnologías audiovisuales para su implementación en espacios públicos, tales como la multimedia. Esta ya cuenta con un tramo recorrido que abarca sistemas y múltiples medios de expresión electrónico, así como virtual. Los proyectos multimedia son capaces de brindar mayores alcances en la generación de emociones e interacción con la audiencia, aplicando así, herramientas de texto, imagen, video y animación, aportando una experiencia más integral.

De este modo, es que las ciudades han sido empleadas como un medio de expresión y de mensajes socioculturales, hechos por artistas, creativos, militantes e incluso regímenes. Sus representaciones las desarrollan en espacios construidos, abiertos y cerrados, públicosy privados, en los que comunican datos, referencias, diálogos, discusiones, sucesos, entre otros argumentos. Por tanto, al igual que consideramos en la publicación "El mural como vehículo narrativo e identidad ciudadana" (2016 p. 3069), la ciudad puede ser el vehículo portavoz de las expresiones del individuo, así como el carácter social que lo identifica como un ser pensante, racional, con emociones, errores, que cambia constantemente, que se mezcla entre diferentes estratos y se compromete a preservar la tolerancia en sus variadas aristas, esto es parte de 
lo que caracteriza al habitante que vive alrededor de un contexto contemporáneo, en una urbe que crece continuamente sin dejar atrás ese hilo histórico que le ha dado origen.

Cabe destacar, la existencia de representaciones religiosas y tradiciones que se llevan a cabo cíclicamente en ciertas zonas o barrios, los cuales se vuelven el escenario para todas estos performances, que ni siempre requieren de herramientas tecnológico-audiovisuales, sino que la misma audiencia es la que produce la interacción y participación a partir de los recursos que le brinda el espacio físico, tanto natural como urbano.

Frente a esta dinámica entre sociedad y tecnologías, el investigador y diseñador Bonsiepe (2007, online) defiende que una política industrializada debería incluir a la industria de la información, para la cual el diseño de información puede prestar servicios esenciales. En este sentido, surgen nuevas temáticas relacionadas al diseño de la comunicación con exigencias cognitivas, tal como la Transmedia, que actúa y se posiciona en este escenario contemporáneo. El nombre de Transmedia y Transmedialidad, dice Orozco (1997, p. 31) que, "durante el 2001, estos dos términos surgieron de la publicidad, de un mundo efímero y plástico; tiempo después tomaron una nueva posición y fueron asimilados por los investigadores y académicos para generar teorías y hasta incluirlos en los programas educativos".

De igual manera, la transmedia dice Jenkins (2008) es "una historia narrativa, un mundo narrativo, tan grande que no cabe en tan solo una plataforma y se expande por distintos canales y formatos, ambos autorreferenciales, construyendo una narrativa envolvente, inmersiva, integradora y participativa". En palabras de Scolari (2013, online), este interpreta a la transmedia como "un tipo de relato en que la historia se desarrolla a través de múltiples medios y plataformas de comunicación y hay una parte de los consumidores que asumen un papel activo en ese proceso de expansión".

En ese mismo camino, Ardini (2018 p. 14-15) defiende a la transmedia "desde la noción de territorio, pero definida no solo desde lo geográfico sino principalmente desde la articulación entre narrativas, tecnologías y participación. (...) Además, cree que todo espacio que sea propicio para que se desarrolle la conversación social es un territorio del transmedia. La experiencia transmedia ocupa los espacios, los transita, los reconstruye, los desarma y vuelve armar, empoderando la ciudad y transformándola en una plataforma narrativa compleja y diversa, probablemente más que cualquier otra que podamos imaginar. La ciudad, como territorio transmedia, está repleta de dispositivos y géneros que se nutren y se hace eco de las voces de quienes la habitan, con sus múltiples lenguajes".

Además de lo anterior, la "narrativa" es uno de los elementos principales del lenguaje transmedia, pues esta permite generar la inquietud de los participantes e identificarse con ellos, hasta el punto de crear un vínculo emocional. La factibilidad de enriquecer la estructura colaborativa es mayor, cuando se ofrece una proximidad con el entorno del usuario o fan, como es nombrado por los teóricos de la narratología. Llevar al fan por un trayecto trazado con elementos que lo envuelvan, significa una capa de niveles que profundizan en la secuencia narrativa.

Otro punto que considerar, enunciado por Galán (2012), son los aspectos técnicos. Si bien, no son características propias de la transmedia, ni tampoco pudieran ser necesarios, pero existen criterios a tomar en cuenta, sobre todo por los guionistas y desarrolladores de proyectos transmedia, tales como, geolocalización, juegos multiplataforma interactivos online/offline, Realidad Aumentada, Mapping bajo la creación de mapas y visualización de datos.

\section{Conclusión}

A lo largo de este texto nos aproximamos a temas relacionados a la arquitectura, al diseño de la información, a las tecnologías, a la sociedad y su relación con los medios de comunicación, así como, a proyectos e iniciativas que buscan la resolución de problemas y procuran el mejoramiento en la calidad de vida de los individuos. De esta forma, confirmamos nuestro entendimiento de que todos los temas aquí desdoblados son parte de los mecanismos transformadores de la presente sociedad contemporánea y de las futuras generaciones.

La Mediatización Urbana, tema central de esta edición editorial, nos permitió explorar en otras áreas de las ciencias humanas, que también trabajan con temáticas afines al desarrollo tecnológico y 
humano. Estas ejemplifican la diversidad existente en los enfoques de interés, necesidades y posibles soluciones en la creación de complejas propuestas proyectuales que se interconectan. Cabe mencionar, que es enriquecedor el haber recorrido a través de las diferentes temáticas aquí planteadas y constatar las similitudes convergentes que estas desarrollan, todas ellas en mayor o menor grado, se apoyan en herramientas tecnológicas, que les permiten realizar configuraciones atrayentes y proponer intervenciones sobre los modelos ya existentes, de tal modo que las configuraciones urbanas y mediáticas repunten en el bienestar social.

\section{REFERENCIAS}

Altamirano, O. (2015). Hombre Bestia entre las calles rosarinas. En F. Irigaray; A. Lovato (Eds). Producciones transmedia de no ficción- Análisis, experiencias y tecnologías. Rosario, Argentina: UNR Editorial. Recuperado de: https://www.academia.edu/22718076/Producciones transmedia de no ficci\%C3\%B3n. An\%C3\%A1lisis experiencias y tecnolog\%C3\%ADas 2015

Altamirano, O; Moura, M. (2016). El mural como vehículo narrativo e identidad ciudadana. 12 P\&D 2016. Congresso Brasileiro de Pesquisa e Desenvolvimento em Design. N. 2 Vol. 9. P. 3069. Recuperado de: http://pdf.blucher.com.br.s3-sa-east-1,amazonaws.com/designproceedings/ped2016/0263.pdf

Altamirano, O; Moura, M. (2017). La ciudad como red y sistema de navegación. Revista Procesos Urbanos. N. 4. Recuperado de: https://revistas.cecar.edu.co/index.php/procesos-urbanos/article/view/349/310

Altamirano, O; Moura, M; Portugal, C. (2018). Transmedia Digital Inclusion. J. Bowen; G. Diprose; N. Lambert; J. Weinel. Electronic Visualisation and the Arts. Recuperado de: https://www.scienceopen.com/ document file/3ca27a2c-8221-4f6d-87f3-e3dc83d1d94c/ScienceOpen/127 Altamirano.pdf

Ardini, C. (2018). Contar (las) historias: manual para experiencias transmedia sociales. Recuperado de: http://transmediacordoba.org.ar/wp-content/uploads/2018/08/ Contar-Ias-historias-Manual-para-experiencias-transmedia-sociales. pdf?fbclid=IwAROnVTYNxjY5jij6VEKCDRzoQtf7F3ZazEHvEhRLj84R4V1abXKvhA1LIcA

Bonsiepe, G. (2011). Design, cultura e sociedade. São Paulo, Brasil: Blucher. p. 58, 61.

Bonsiepe, G. (2007). Design e democracia. Revista Brasileira de Design. Ensaios. Número: 2. Recuperado de: http://www.agitprop.com.br/index.cfm?pag=ensaios det\&id=13\&Titulo=ensaios

Cárdenas, D; Camargo, F. (2016). Las smart cities, la comunicación y el diseño: un reto de la sociedad del conocimiento. Revista Virtualmente, 4 (2). P. 49. Recuperado de: https://journal.universidadean.edu.co/ index.php/vir/article/view/1794/1698

De la Cruz Severiche, Z., y Guevara Guevara, O. A. (2015). La gestión urbana en las ciudades inteligentes. Procesos Urbanos, 2(2), 73-83. https://doi.org/10.21892/2422085X.8

Falzoni, R. (2020). O que os entregadores de app querem com a greve?. Bike é Legal. Recuperado de: https://www.youtube.com/watch?v=|BfopGwFgol

Galán, J. (2012). La transmedialidad: una nueva gramática para el sujeto complejo. Portal Comunicación. Recuperado de: http://www.portalcomunicacion.com/lecciones.asp?aut=81 
Gassmann, O; Böhm, J; Palmié, M. (2019). Smart Cities. Reino Unido: Edición de Kindle. (pp. 6, 29).

Jenkins, H. (2008). Convergence Culture, La Cultura de la Convergencia de los Medios de Comunicación, Barcelona: Paidós.

Lévy, P. (1999). Cibercultura. São Paulo, Brasil: Editora 34.

Martel, F. (2015). Smart. O que você não sabe sobre a Internet. Rio de Janeiro: Civilização Brasileira.

Martel, F. (2014). Global gay. Barcelona: Penguin Random House Grupo Editorial España. Edición de Kindle.

Moura, M. (2003). O design de hipermídia. São Paulo: Comunicação e semiótica PUC/SP.

McLuhan, M. (1964). Os meios de comunicação como extensões do homem (understanding media). São Paulo, Brasil: Editora Cultrix. Recuperado de: https://edisciplinas.usp.br/pluginfile.php/352425/ mod resource/content/1/MCLUHAN\%2C\%20Marshall\%20-\%200s\%20Meios\%20de\%20 Comunicac\%CC\%A7a\%CC\%830\%20como\%20Extenso\%CC\%83es\%20do\%20Homem.pdf

Orozco, G. (1997). La Investigación de la comunicación dentro y fuera de América Latina. Tendencias, perspectivas y desafíos del estudio de los medios. La Plata, Argentina: Universidad Nacional de La Plata, Ediciones de Periodismo y Comunicación.

Rüdiger, F. (2002). Elementos para a crítica da cibercultura. São Paulo: Hacker Editores.

Scolari, C. (2013). Narrativas Transmedia: cuando todos los medios cuentan. Barcelona: Centro Libros Papf.

Yung, F. (2019). Un colorido acuario urbano irrumpe en la jungla de cemento brasileña. Ultima Hora. EFE Nayara Batschke. Recuperado de: https://www.ultimahora.com/un-colorido-acuario-urbano-irrumpela-jungla-cemento-brasilena-n2851341.html 